Regular research paper

\title{
Supercritical fluid extraction as a preparation method for mass spectrometry of dried blood spots
}

\author{
Atsuki Matsubara $^{\mathrm{a}}$, Yoshihiro Izumi ${ }^{\mathrm{b}}$, Shin Nishiumi ${ }^{\mathrm{a}}$, Makoto Suzuki $^{\mathrm{a}}$, Takeshi Azuma ${ }^{\mathrm{a}}$, \\ Eiichiro Fukusaki ${ }^{\mathrm{b}}$, Takeshi Bamba ${ }^{\mathrm{b}^{*}}$, Masaru Yoshida $\mathrm{a}^{\mathrm{a}, \mathrm{c}, \mathrm{d}^{* *}}$
}

aDivision of Gastroenterology, Department of Internal Medicine, Kobe University Graduate School of Medicine, 7-5-1, Kusunoki-cho, Chuo-ku, Kobe, Hyogo 650-0017, Japan

${ }^{b}$ Department of Biotechnology, Graduate School of Engineering, Osaka University, 2-1 Yamadaoka, Suita, Osaka 565-0871, Japan

cThe Integrated Center for Mass Spectrometry, Kobe University Graduate School of Medicine, 7-5-1, Kusunoki-cho, Chuo-ku, Kobe, Hyogo 650-0017, Japan

dDivision of Metabolomics Research, Department of Internal Medicine, Kobe University Graduate School of Medicine, 7-5-1, Kusunoki-cho, Chuo-ku, Kobe, Hyogo 650-0017, Japan

\section{Correspondent footnote:}

*Takeshi Bamba, Ph.D.

Department of Biotechnology, Graduate School of Engineering, Osaka University, 2-1 Yamadaoka, Suita, Osaka 565-0871, Japan

Tel.: +81-6-6879-7418; Fax: +81-6-6879-7418; E-mail: bamba@bio.eng.osaka-u.ac.jp 
**Masaru Yoshida M.D., Ph.D.

Division of Metabolomics Research, Division of Gastroenterology, The Integrated Center for Mass Spectrometry, Kobe University Graduate School of Medicine, 7-5-1, Kusunoki-cho, Chuo-ku, Kobe, Hyogo 650-0017, Japan

Tel.: +81-78-382-6305; Fax:+81-78-382-6309; E-mail: myoshida@med.kobe-u.ac.jp 


\begin{abstract}
The potential of supercritical fluid extraction (SFE) as a preparation method for mass spectrometry of dried blood spots (DBS) was examined. SFE is generally used for the extraction of hydrophobic compounds, but hydrophilic metabolites such as amino acids, amines, and nucleic-acid-related metabolites could be extracted by adding a low level of methanol as a modifier. Under the optimized conditions, over 200 metabolites were detected from a dried serum spot, of which over 160 metabolites could be analyzed stably (RSD $<20 \%$ ). These results show that SFE is an effective extraction method of metabolites with a wide range of polarity in DBS.
\end{abstract}

\title{
Keywords
}

Supercritical fluid extraction (SFE); Dried blood spot (DBS); Mass spectrometry (MS); Metabolite; Metabolome analysis 


\section{Abbreviations}

DBS, dried blood spot; MS, mass spectrometry; TDM, therapeutic drug monitoring; SFE, supercritical fluid extraction; $\mathrm{SCCO}_{2}$, supercritical carbon dioxide; LC/MS/MS, liquid chromatography/tandem mass spectrometry; BHT, dibutylhydroxytoluene; DSS, dried serum spot; DWBS, dried whole blood spot; PC, phosphatidylcholine; PE, phosphatidylethanolamine; OSE, organic solvent extraction; SFC, supercritical fluid chromatography 


\section{Introduction}

There are many advantages of dried blood spot (DBS) sampling compared with the conventional blood sampling method, including lower sample consumption and easier handling [1-4]. The former advantage enables easier application of blood sampling to newborns and reduction of the use of experimental animals. The latter contributes to reduce costs for transport and the preservation of samples.

Owing to these advantages, DBS has been applied for newborn mass screening $[5,6]$ and for HIV tests [7]. Furthermore, combined with mass spectrometry (MS), the application of DBS is being extended to therapeutic drug monitoring (TDM) [8-10], pharmacokinetic study $[11,12]$, and metabolome analysis $[13,14]$. Although there are several problems about false positive detection such as cross-talk or in-source decay, MS is a highly sensitive and selective detection method, and is free from the problem of cross-reactivity $[15,16]$, which is a major defect of an indirect detection method that uses antibody response. Furthermore, MS can target multiple metabolites at one analysis [17]. Generally, MS's drawback is low quantitative capability due to ion suppression, but this problem can be solved by using stable isotopes of target compounds [18]. The combination of DBS with MS is a promising strategy for biochemical analysis.

Supercritical fluid extraction (SFE) is an extraction method that has features such as rapidity, high selectivity, and low solvent consumption $[19,20]$. It is generally used for the extraction of hydrophobic compounds such as fat-soluble vitamins [19,21], carotenoids [22], fatty acids [23], and aliphatic hydrocarbons[24], owing to the high hydrophobicity of supercritical carbon dioxide $\left(\mathrm{SCCO}_{2}\right)$. In our previous study [25], we applied SFE as an extraction method for phospholipids in DBS. However, other metabolites including hydrophilic metabolites were not targeted in that study. Recently, on the other hand, it has been reported that hydrophilic compounds such as amino acids could be extracted from crops by SFE with a relatively large amount of modifier [26,27]. This suggests that SFE can target 
the wide variety of metabolites contained in DBS.

In this study, SFE's potential as a preparation method for MS using DBS was evaluated. To specify SFE's applicable range of polarity, the extracts obtained from SFE of DBS were analyzed by liquid chromatography/tandem mass spectrometry (LC/MS/MS), which target lipids (phospholipids, fatty acids, acylcarnitines, bile acids) as well as hydrophilic compounds (amino acids, amines, nucleic-acid-related metabolites). The extraction conditions were optimized and the extraction efficiency of SFE was compared to that of the commonly used organic solvent extraction to characterize SFE as a DBS extraction method.

\section{Experimental}

\subsection{Chemicals}

$\mathrm{CO}_{2}$ used for SFE was purchased from Iwatani Corporation (Tokyo, Japan). As an extraction medium, methanol (HPLC grade) was obtained from Nacalai Tesque Inc. (Kyoto, Japan). Acetone and dibutylhydroxytoluene (BHT) used in the extraction procedure were obtained from Wako Pure Chemical Industries Ltd. (Osaka, Japan). For LC/MS/MS, water and acetonitrile (LC/MS grade) were purchased from Wako Pure Chemical Industries Ltd. and methanol (LC/MS grade) was obtained from Kanto Chemical Co. Inc. (Tokyo, Japan). Formic acid (LC/MS grade, Wako Pure Chemical Industries Ltd.) and ammonium acetate (1 M solution, HPLC grade, Wako Pure Chemical Industries Ltd.) were used as additives for the mobile phase. 2-Bromohypoxanthine purchased from Sigma-Aldrich (MO, USA) and dilauroylphosphatidylcholine (PC 12:0-12:0) purchased from Avanti Polar Lipids (AL, USA) were used as internal standards.

\subsection{Samples}

BondElut DMS card (Agilent Technologies, CA, USA) was punched using a 
dedicated hole puncher (3 mm, I.D.). As an antioxidant, $30 \mu \mathrm{l}$ of $0.5 \%$ BHT (Wako Pure Chemical Industries Ltd.) in acetone (HPLC grade, Wako Pure Chemical Industries) was added to the hollowed out pieces [28]. After drying, $3 \mu$ l of human serum or whole blood from a healthy subject, including $30 \mu \mathrm{M}$ 2-bromohypoxanthine and 2.4 $\mu \mathrm{M}$ PC 12:0-12:0 as internal standards, was dropped on the punched pieces and dried for over two hours at room temperature. These samples are referred to as dried serum spots (DSS) and dried whole blood spots (DWBS), respectively, in this article.

\subsection{Extraction conditions}

In this study, a prototype SFE system made by Shimadzu Corporation (Kyoto, Japan) was used as an SFE instrument. Extraction was performed in dynamic mode. For the collection of extracts, the bubbling method was used. Time for extraction was 5 min because $5 \mathrm{~min}$ was sufficient for minimum flow rate condition in this study (methanol $0.3 \mathrm{ml} / \mathrm{min}$ ). Other extraction conditions are shown in the main text and the figure legends. Methods for organic solvent extraction were as follows: $300 \mu \mathrm{l}$ of methanol was added to the dried blood spot and left to stand for an hour. After centrifugation $\left(15,000 \mathrm{~g}, 4^{\circ} \mathrm{C}, 5 \mathrm{~min}\right)$, the supernatant was collected into a new tube. The collected extracts were dried by centrifugal concentration, and reconstituted with $50 \mu 1$ of water (Kanto Chemical) for hydrophilic metabolite analysis or methanol for lipid analysis.

\subsection{Analytical conditions}

Analyses were carried out using a Nexera LC system (Shimadzu Corp.) equipped with two LC-30AD pumps, a DGU-20As degasser, a SIL-30AC auto sampler, a CTO-20AC column oven, and a CBM-20A control module, coupled with an LCMS-8040 triple quadrupole mass spectrometer (Shimadzu Corp.). The hydrophilic metabolites were separated using a pentafluorophenyl column (Discovery HS F5, 150 x $2.1 \mathrm{~mm}, 3 \mu \mathrm{m}$, SUPELCO, PA, 
USA) with a guard column (20 mm x $2.1 \mathrm{~mm}, 3 \mu \mathrm{m})$, while lipids were separated using an octadecylsilylated silica column (Inertsustain C18, 100 x $2.1 \mathrm{~mm}, 3 \mu \mathrm{m}$, GL Sciences, Tokyo, Japan) with a guard column $(10 \times 3 \mathrm{~mm}, 5 \mu \mathrm{m})$. The mobile phase for hydrophilic metabolites was constituted with A: $0.1 \%$ formic acid in water and B: acetonitrile. The flow rate was $0.3 \mathrm{ml} / \mathrm{min}$ and the column oven temperature was $40^{\circ} \mathrm{C}$. The gradient program for mobile phase $\mathrm{B}$ was as follows: $0 \mathrm{~min}, 0 \% ; 7 \mathrm{~min}, 0 \%$; $20 \mathrm{~min}, 40 \% ; 20.1 \mathrm{~min}, 100 \%$; 25 min, 100\%; $25.1 \mathrm{~min}, 0 \%$; and $35 \mathrm{~min}, 0 \%$. The mobile phase for lipids consisted of A: 20 $\mathrm{mM}$ ammonium acetate in water and $\mathrm{B}$ : methanol. The flow rate was $0.4 \mathrm{ml} / \mathrm{min}$ and the column oven temperature was $40^{\circ} \mathrm{C}$. The gradient program for mobile phase $\mathrm{B}$ was as follows: $0 \mathrm{~min}, 80 \%$; $13 \mathrm{~min}, 98 \%$; $30 \mathrm{~min}, 98 \%$; $30.1 \mathrm{~min}, 80 \%$; and $35 \mathrm{~min}, 80 \%$. The target metabolites of these systems are shown in Supplementary Table 1. Typical MRM chromatograms of these metabolites are shown in Supplementary Fig. 1. Hydrophilic metabolites were evaluated by peak area whereas lipids were evaluated by peak height because some lipid isomers could not be separated completely.

\section{Result and discussion}

\subsection{Optimization of SFE conditions}

First, basic conditions for extraction were investigated using methanol as a modifier. Direct collection caused splatter of the extracts. Therefore, extracts contained in the mixture of $\mathrm{CO}_{2}$ and methanol were bubbled and trapped in methanol, at the bottom of a test tube. In order to determine what kind of metabolites can be extracted using this system, extraction of a dried serum spot (DSS) was performed under the intermediate conditions (pressure, 20 $\mathrm{MPa}$; temperature, $30^{\circ} \mathrm{C}$; methanol, $0.6 \mathrm{ml} / \mathrm{min} ; \mathrm{CO}_{2}, 2.4 \mathrm{ml} / \mathrm{min}$ ) and the extracts were analyzed under two different LC/MS/MS conditions that target either lipid or hydrophilic metabolites (data not shown). As a result, in addition to the formerly reported phosphatidylcholines (PC) and phosphatidylethanolamines (PE) [25], hydrophobic 
metabolites such as acylcarnitines and bile acids were detected by analysis with the extracts. Furthermore, many hydrophilic metabolites such as amino acids, nucleic-acid-related metabolites, and betaines (carnitine, choline, etc.) were also extracted by SFE. These results clearly show that DSS-SFE samples contain the metabolites with a variety of polarities.

Next, parameters of SFE such as temperature, pressure, and modifier were optimized by comparing extraction efficiency of the metabolites representative of each metabolite class under the testing conditions (amino acids, isoleucine; nucleic-acid-related metabolites, hypoxanthine; betaines, choline; other hydrophilic metabolites, creatinine; bile acids, glycodeoxycholic acid; free fatty acids, linoleic acid; PC, PC 18:0-18:2/18:1-18:1; PE, PE 18:0-18:2/18:1-18:1 using DSS as samples. First, extraction temperature $\left(30,50,80^{\circ} \mathrm{C}\right)$ was investigated under the following conditions: $\mathrm{CO}_{2}, 2.4 \mathrm{ml} / \mathrm{min}$; methanol, $0.6 \mathrm{ml} / \mathrm{min}$; pressure, $20 \mathrm{MPa}$ : time for extraction, $5 \mathrm{~min}$. Although $80^{\circ} \mathrm{C}$ was the only condition that met the supercritical conditions among these conditions [29], there was no dramatic improvement in extraction efficiency (Fig. 1A). These results indicate that whether the extraction medium was supercritical or subcritical state did not affect much on extraction efficiency. Actually, the extraction efficiency tended to decrease as the temperature increased. In general, the density of $\mathrm{SCCO}_{2}$ decreases when temperature is increased. Therefore, it stands to reason that the solvent power decreased as temperature increased [30]. In addition, the breakdown of the analytes was another possible reason for this result. Secondly, extraction pressure $(10,20,30$ $\mathrm{MPa}$ ) was investigated under the following conditions: $\mathrm{CO}_{2}, 2.4 \mathrm{ml} / \mathrm{min}$; methanol, 0.6 $\mathrm{ml} / \mathrm{min}$; temperature, $30^{\circ} \mathrm{C}$; time for extraction, $5 \mathrm{~min}$. Generally, the increase of pressure makes the density and the solvent power of $\mathrm{SCCO}_{2}$ increase [30]. However, as a result, pressure did not markedly affect the extraction efficiency (Fig. 1B). There are two possible reasons for these results. The first is that the time for extraction was more than sufficient for this sample volume; therefore, the solvent power did not affect the results much. The second reason is that, in this subcritical condition, pressure has a minimal effect on extraction 
efficiency. Finally, the effect of solvent constitution was investigated (Fig. 2). Temperature and pressure were fixed at $30^{\circ} \mathrm{C}$ and $20 \mathrm{MPa}$, respectively, and various $\mathrm{SCCO}_{2} /$ methanol compositions $\left(0.3 \mathrm{ml} / \mathrm{min}\right.$ methanol, $1.2 \mathrm{ml} / \mathrm{min}$ methanol, $0.9 \mathrm{ml} / \mathrm{min} \mathrm{SCCO}_{2}$ and $0.3 \mathrm{ml} / \mathrm{min}$ methanol, $1.8 \mathrm{ml} / \mathrm{min} \mathrm{SCCO}_{2}$ and $0.3 \mathrm{ml} / \mathrm{min}$ methanol) were investigated. The results of 0.3 $\mathrm{ml} / \mathrm{min}$ methanol only were nearly the same as those of $1.2 \mathrm{ml} / \mathrm{min}$ methanol only. This indicates that 5 min was enough time for DSS extraction. The effect of $\mathrm{CO}_{2}$ addition can be estimated using $0.3 \mathrm{ml} / \mathrm{min}$ methanol with $0,0.9$, and $1.8 \mathrm{ml} / \mathrm{min} \mathrm{SCCO}_{2}$. As a result, the more $\mathrm{CO}_{2}$ added, the higher the extraction efficiency of lipids tends to be; on the other hand, that of hydrophilic metabolites has a tendency to be lower. These results indicate that, at least to a certain extent, there is an effect of $\mathrm{SCCO}_{2}$ on hydrophobicity. However, the difference is not drastic and it seemed that methanol is a dominant factor in the DBS extraction process.

\subsection{Comparison of SFE with conventional organic solvent extraction}

Next, SFE was compared with organic solvent extraction (OSE), which is the commonly used preparation method for DBS. From the above-mentioned results, extraction conditions were determined as follows: pressure, $20 \mathrm{MPa}$; temperature, $30^{\circ} \mathrm{C}$; methanol, 0.3 $\mathrm{ml} / \mathrm{min}$; and $\mathrm{CO}_{2}, 1.8 \mathrm{ml} / \mathrm{min}$; time for extraction, $5 \mathrm{~min}$. For analysis targeting metabolites with wide range of polarity, it is difficult to determine the only extraction condition which is best for all target metabolites. In this study, therefore, methanol that was the same solvent for modifier of SFE was chosen as organic solvent to minify the difference in extraction condition for easier understand of the results. For SFE conditions, lower $\mathrm{CO}_{2}$ flow rate was better for hydrophilic metabolites, while higher $\mathrm{CO}_{2}$ flow rate was better for lipids. In this study, with a view to construct an online coupling to supercritical fluid chromatography (SFC), a single extraction condition was chosen. For online SFE system, in order to trap metabolites in a trap column, low modifier concentration was desired. Therefore, the highest $\mathrm{CO}_{2}$ flow rate $(1.8 \mathrm{ml} / \mathrm{min})$ was chosen. As a result, 37 hydrophilic metabolites including 
amino acids, betaines, nucleic acids, and 195 lipids including (lyso)phosphatidylcholines, (lyso)phosphatidylethanolamines, bile acids, and acylcarnitines were detected from DSS-SFE samples, whereas 40 hydrophilic metabolites and 185 lipids were detected from DSS-OSE (Table 1, Suppl. Table 1). These results clearly show that SFE with the modifier methanol had no disadvantages in hydrophilic metabolite extraction. The number of stably analyzed (RSD $<20 \%$ ) metabolites from SFE samples was 17 hydrophilic metabolites and 144 lipids. Many of the metabolites that showed significantly higher intensity by SFE were phospholipids and fatty acids, some of which were detected only from SFE samples (Table 2, Suppl. Table 1). These results indicate that the extraction efficiency of hydrophobic metabolites was enhanced by $\mathrm{SCCO}_{2}$. Dried whole blood spots (DWBS) were extracted in the same way. It is difficult to store whole blood samples. Dried spot analysis can be one of the solutions to this problem. As a result, many PE were detected only from DWBS (Table 3, Suppl. Table 1). PE are one of the main phospholipids in the blood cells that are removed in the serum sample [31]. These results suggest that DWBS are useful samples for the detection of the metabolites that cannot be targeted in the case of serum analysis.

\section{Conclusion}

In this study, the potential of SFE as a DBS preparation method was examined. Generally, SFE has been considered as effective for hydrophobic compound extraction. However, the addition of methanol as a modifier significantly improved SFE's applicable range of polarity, and there were no problems in hydrophilic metabolite extraction from DSS or DWBS. As a result, over 200 metabolites were detected by LC/MS/MS, of which over 160 metabolites were analyzed stably (RSD $<20 \%$ ). It was shown that the addition of $\mathrm{CO}_{2}$ improved the extraction efficiency of hydrophobic metabolites.

Furthermore, it is easy to connect SFE with analytical instruments and to construct an online extraction system. Actually, we have developed a high-throughput screening system 
for phospholipids using supercritical fluid chromatography (SFC)/MS coupled with online SFE [25]. For online extraction, the trapping of target compounds before the separation step is essential, but the most difficult point to be investigated. In SFC, organic solvents increase the power for elution. Therefore, the trapping of target compounds cannot be achieved if a high level of organic solvent is used for the extraction step. This study revealed that SFE requires a low level of methanol to extract hydrophilic metabolites and showed the possibility that many hydrophilic metabolites can be the targets of online SFE-SFC/MS/MS. Several studies were conducted to achieve online extraction of multiple metabolites, and they did not use chromatographic separation before MS analysis [32,33]. These systems cannot separate isomers and cannot avoid ion suppression, which are the main defects of MS. Online SFE-SFC/MS can solve these problems. SFE of DBS, coupled with SFC/MS, is expected to be an ideal analytical technique for TDM, pharmacokinetic study, and multiple biomarker quantification.

\section{Acknowledgement}

Sincere appreciation is expressed to Shimadzu Corporation for valuable technical support. This study was supported in part by a grant from the 'Young Researchers Training Program for Promoting Innovation' run by the Special Coordination Fund for Promoting Science and Technology from the Ministry of Education, Culture, Sports, Science, and Technology of Japan (MEXT) [A.M., T.A.]; a Grant-in-Aid for Research Activity Start-up [A.M.], a Grant-in-Aid for Scientific Research (B) [M.Y.], a Grant-in-Aid for Exploratory Research [M.Y.] and a Grant-in-Aid for Young Scientists (A) [T. B.] from MEXT; and a grant from the Development of Systems and Technology for Advanced Measurement and Analysis Project (JST) [Y.I., M.S., T.B., M.Y.]. 


\section{References}

[1] W. Li, F.L.S. Tse, Biomed. Chromatogr. 24 (2010) 49.

[2] B.G. Keevil, Clin. Biochem. 44 (2011) 110.

[3] S.P. Parker, W.D. Cubitt, J. Clin. Pathol. 52 (1999) 633.

[4] P. a Demirev, Anal. Chem. 85 (2013) 779.

[5] T.H. Zytkovicz, E.F. Fitzgerald, D. Marsden, C. a Larson, V.E. Shih, D.M. Johnson, a W. Strauss, a M. Comeau, R.B. Eaton, G.F. Grady, Clin. Chem. 47 (2001) 1945.

[6] J.H. Miller, P. a Poston, H.T. Karnes, J. Chromatogr. B. Analyt. Technol. Biomed. Life Sci. 903 (2012) 142.

[7] I.J.M. Snijdewind, J.J. a van Kampen, P.L. a Fraaij, M.E. van der Ende, A.D.M.E. Osterhaus, R. a Gruters, Antiviral Res. 93 (2012) 309.

[8] J. van der Heijden, Y. de Beer, K. Hoogtanders, M. Christiaans, G.J. de Jong, C. Neef, L. Stolk, J. Pharm. Biomed. Anal. 50 (2009) 664.

[9] T. Koal, H. Burhenne, R. Römling, M. Svoboda, K. Resch, V. Kaever, Rapid Commun. Mass Spectrom. 19 (2005) 2995.

[10] G. la Marca, S. Malvagia, L. Filippi, P. Fiorini, M. Innocenti, F. Luceri, G. Pieraccini, G. Moneti, S. Francese, F.R. Dani, R. Guerrini, J. Pharm. Biomed. Anal. 48 (2008) 1392.

[11] J. Déglon, A. Thomas, Y. Daali, E. Lauer, C. Samer, J. Desmeules, P. Dayer, P. Mangin, C. Staub, J. Pharm. Biomed. Anal. 54 (2011) 359.

[12] Z. Shen, P. Kang, S. V Rahavendran, J. Pharm. Biomed. Anal. 67-68 (2012) 92.

[13] F. Michopoulos, G. Theodoridis, C.J. Smith, I.D. Wilson, J. Proteome Res. 9 (2010) 3328.

[14] S.T. Kong, H.-S. Lin, J. Ching, P.C. Ho, Anal. Chem. 83 (2011) 4314.

[15] C. Staatz, P. Taylor, S. Tett, Ther. Drug Monit. (2002) 607. 
[16] P. Lutter, M.-C. Savoy-Perroud, E. Campos-Gimenez, L. Meyer, T. Goldmann, M.-C. Bertholet, P. Mottier, A. Desmarchelier, F. Monard, C. Perrin, F. Robert, T. Delatour, Food Control 22 (2011) 903.

[17] D.R. Koop, L. a. Bleyle, M. Munar, G. Cherala, A. Al-Uzri, J. Chromatogr. B 926 (2013) 54.

[18] C. Birkemeyer, A. Luedemann, C. Wagner, A. Erban, J. Kopka, Trends Biotechnol. 23 (2005) 28 .

[19] C. Turner, J.W. King, L. Mathiasson, J. Chromatogr. A 936 (2001) 215.

[20] M. Herrero, J. a Mendiola, A. Cifuentes, E. Ibáñez, J. Chromatogr. A 1217 (2010) 2495.

[21] A. De Lucas, E.M. De, J. Rinco, 22 (2002) 221.

[22] M. Careri, L. Furlattini, a Mangia, M. Musc, E. Anklam, a Theobald, C. von Holst, J. Chromatogr. A 912 (2001) 61.

[23] X. Cao, Y. Ito, J. Chromatogr. A 1021 (2003) 117.

[24] M.O. Punín Crespo, M. a Lage Yusty, Ecotoxicol. Environ. Saf. 64 (2006) 400.

[25] T. Uchikata, A. Matsubara, E. Fukusaki, T. Bamba, J. Chromatogr. A (2012).

[26] E. Arnáiz, J. Bernal, M.T. Martín, M.J. Nozal, J.L. Bernal, L. Toribio, J. Chromatogr. A 1250 (2012) 49.

[27] A. Erkucuk, I.H. Akgun, O. Yesil-Celiktas, J. Supercrit. Fluids 51 (2009) 29.

[28] K. Ichihar, K. Waku, C. Yamaguchi, K. Saito, A. Shibahara, S. Miyatani, K. Yamamoto, Lipids 37 (2002) 523.

[29] J.B. Crowther, J.D. Henion, Anal. Chem. 57 (1985) 2711.

[30] E. Reverchon, I. De Marco, J. Supercrit. Fluids 38 (2006) 146.

[31] R.M. Dougherty, C. Galli, A. Ferro-Luzzi, J.M. Iacono, Am. J. Clin. Nutr. 45 (1987) 443. 
[32] A. Thomas, J. Déglon, S. Lenglet, F. Mach, P. Mangin, J.-L. Wolfender, S. Steffens, C. Staub, Anal. Chem. 82 (2010) 6687.

[33] J. Déglon, A. Thomas, A. Cataldo, P. Mangin, C. Staub, J. Pharm. Biomed. Anal. 49 (2009) 1034. 


\section{Figure captions}

Fig. 1. Effects of temperature (A) and pressure (B) for SFE of various metabolites in dried serum spots (DSS). DSS ( $\mathrm{n}=5)$ were extracted under conditions of various temperatures $\left(30^{\circ} \mathrm{C}, 50^{\circ} \mathrm{C}, 80^{\circ} \mathrm{C}\right)$ and pressures $(10 \mathrm{MPa}, 20 \mathrm{MPa}, 30 \mathrm{MPa})$, and then analyzed by LC/MS/MS. The value is shown as the relative peak height (for hydrophilic metabolites) / area (for lipids) to that at $30^{\circ} \mathrm{C}(\mathrm{A})$ or that at $20 \mathrm{MPa}(\mathrm{B})$. Other conditions: pressure for (A), $20 \mathrm{MPa}$; temperature for $(\mathrm{B}), 30^{\circ} \mathrm{C} ; \mathrm{CO}_{2}$ flow rate, $2.4 \mathrm{ml} / \mathrm{min}$; methanol flow rate, 0.6 $\mathrm{ml} / \mathrm{min}$; time for extraction, $5 \mathrm{~min}$.

Fig. 2. Effect of solvent constitution for SFE of various metabolites in dried serum spots (DSS). DSS ( $\mathrm{n}=5)$ were extracted under various $\mathrm{SCCO}_{2} /$ methanol compositions $(0.3 \mathrm{ml} / \mathrm{min}$ methanol, $1.2 \mathrm{ml} / \mathrm{min}$ methanol, $0.9 \mathrm{ml} / \mathrm{min} \mathrm{SCCO}_{2}$ and $0.3 \mathrm{ml} / \mathrm{min}$ methanol, $1.8 \mathrm{ml} / \mathrm{min}$ $\mathrm{SCCO}_{2}$ and $0.3 \mathrm{ml} / \mathrm{min}$ methanol) and analyzed by LC/MS/MS. The value is shown as the relative peak height (for hydrophilic metabolites) / area (for lipids) to that of $\mathrm{CO}_{2}$ at $0 \mathrm{ml} / \mathrm{min}$, methanol at $1.2 \mathrm{ml} / \mathrm{min}$. Other conditions: temperature, $30^{\circ} \mathrm{C}$; pressure, $20 \mathrm{MPa}$; time for extraction, 5 min. 


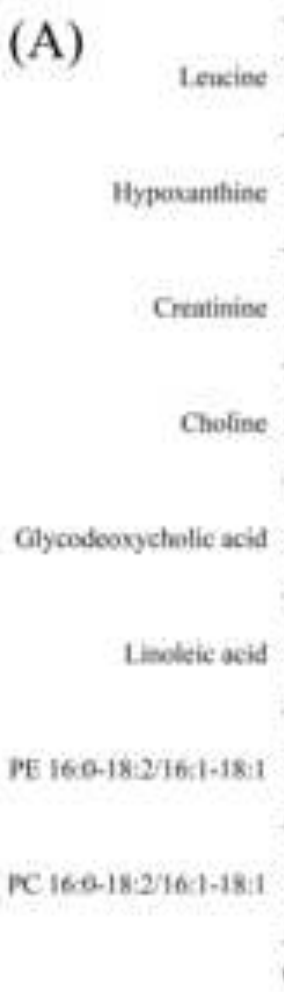

(A) Lencine
Hyposanthine
Crentinine
Choline
Glycodeoxycholic acid
Linoleic acid

A) Lencine
Hyposanthine
Crentinine
Chotine
Liticoleic acid
E i6o-18:216:1-18:1

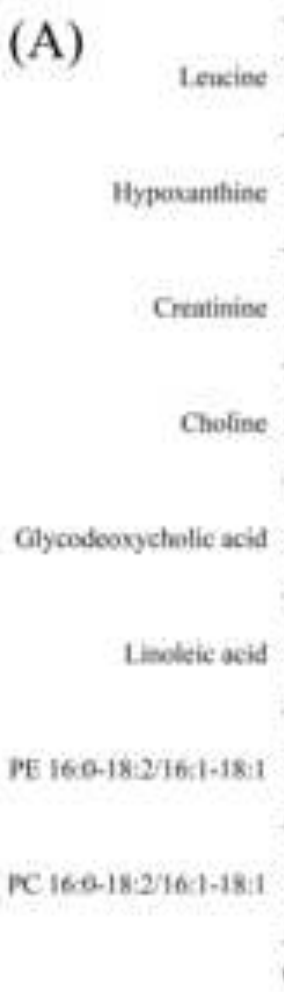

(A) Lencine
Hyposanthine
Cratimine
Choline
Glycodeoxycholic acid
Linoleic acid
PE I6-0-18:216:1-18:1

\section{2}

A) Lencine
Hyposanthine
Crentinine
Chotine
Liticoleic acid
E i6o-18:216:1-18:1
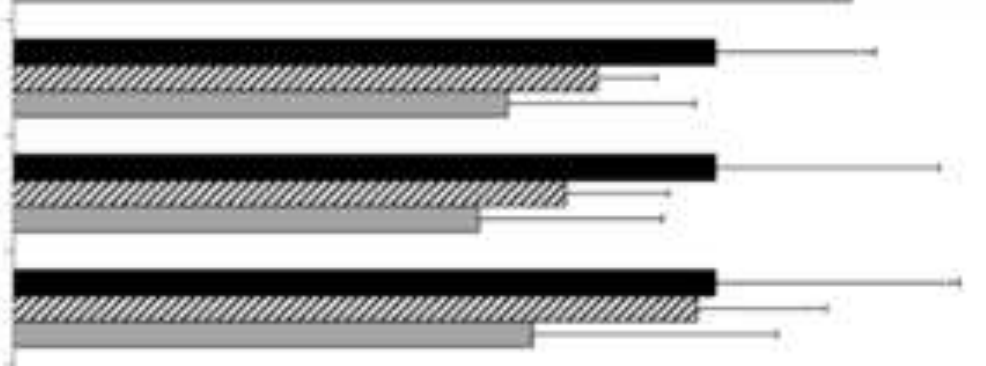

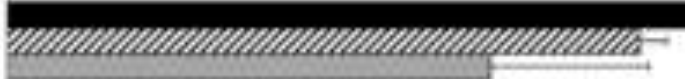
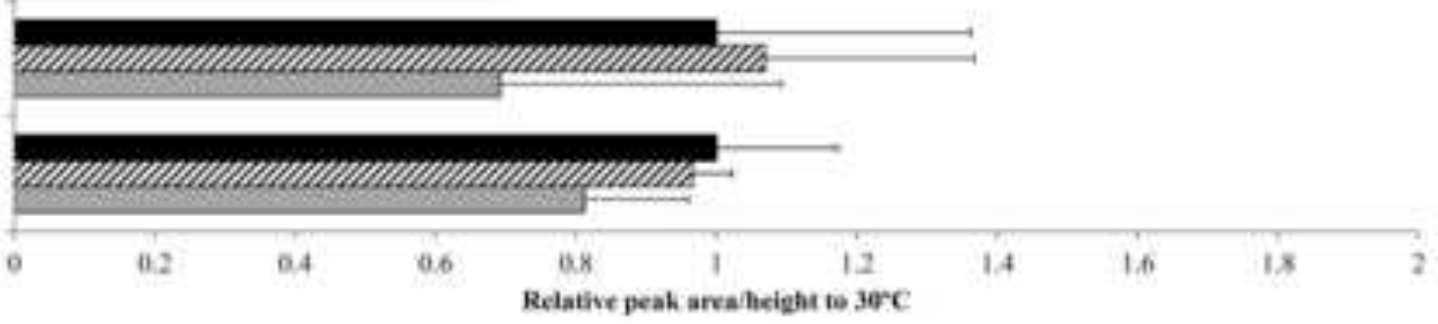

(B)

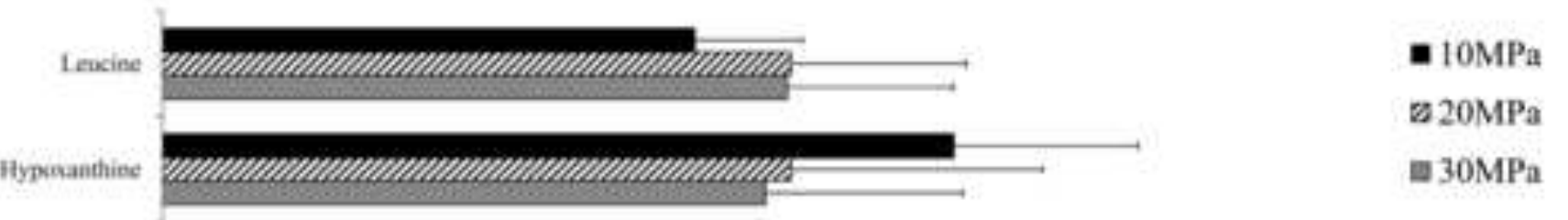

Choline

Glycodeongcholic acid

Limoleic acid

PE 160-18:2/6:1-18:1

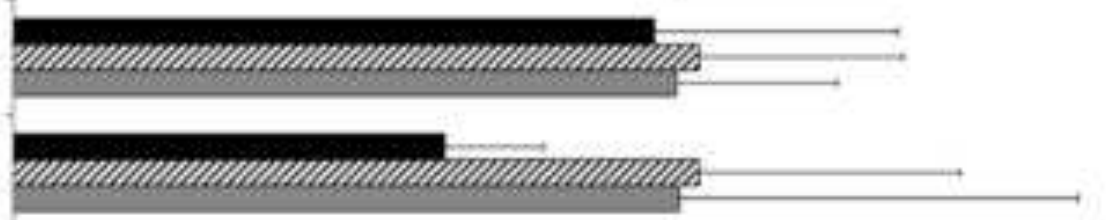

- $30^{\circ} \mathrm{C}$ 2. $50^{\circ} \mathrm{C}$ $\square 80^{\circ} \mathrm{C}$
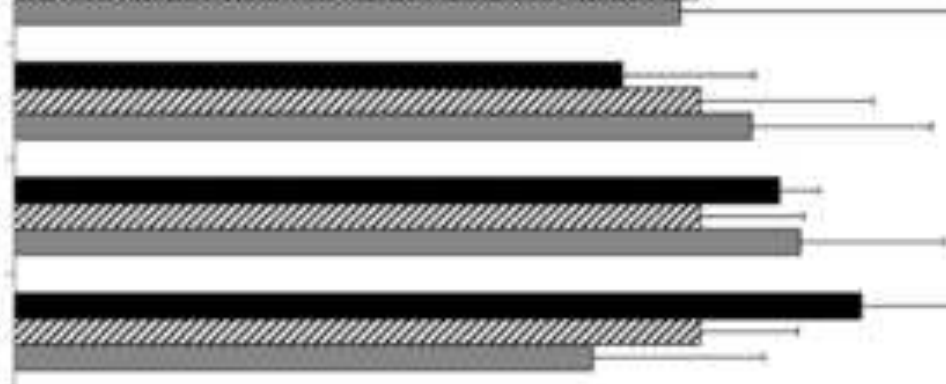

AC 16-0-18:216:1-18:1

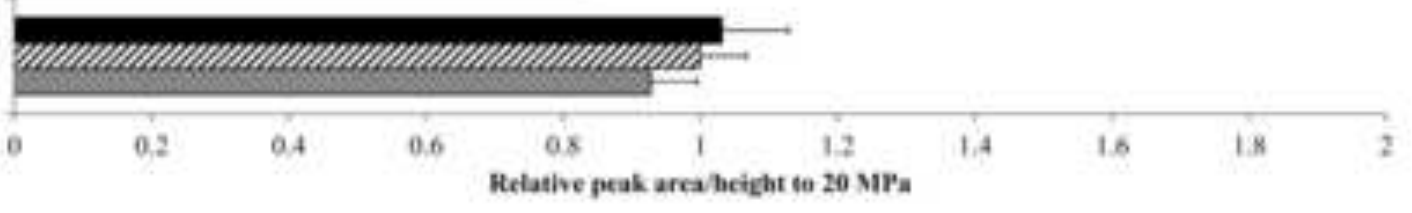

Figure1

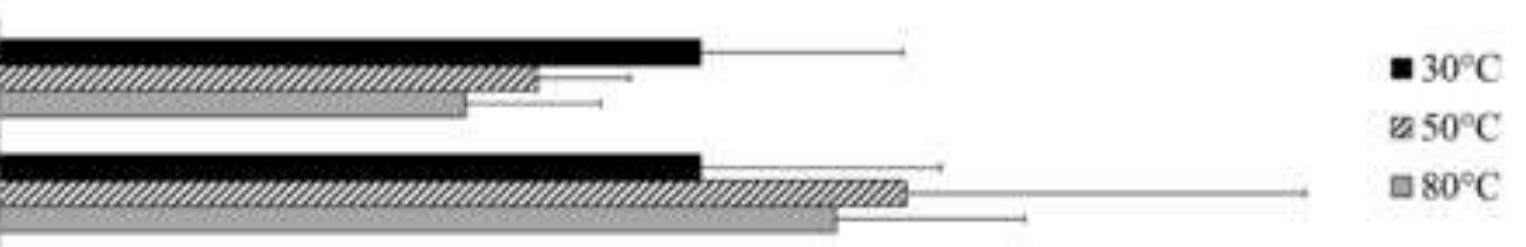

Relative peah arealteight to $30 \mathrm{C}^{\circ} \mathrm{C}$

(10.0.

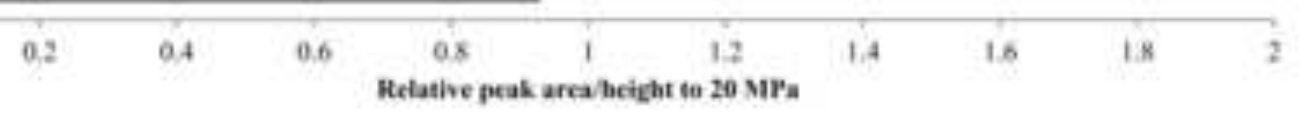


Table 1

Number of metabolites detected from dried blood spot extracted by supercritical fluid extraction (SFE) o

\begin{tabular}{lcccc}
\hline & \multicolumn{2}{c}{ Cation } & \multicolumn{2}{c}{ Lipids } \\
\cline { 2 - 5 } & Detected & RSD $<20$ & Detected & RSD $<20$ \\
\hline SFE & 37 & 17 & 195 & 144 \\
OSE & 40 & 24 & 185 & 127 \\
\hline
\end{tabular}


Table 2

List of metabolites whose peak intensity showed a significant difference between supercritica

\begin{tabular}{ll}
\hline Significantly high in SFE samples & Significantly high in OSE samples \\
\hline LPC 14:0 $(s n-1)$ & Glycine \\
PC 16:0e-16:0 & Guanosine \\
PC 16:0-18:2/16:1-18:1 & Hypoxanthine \\
PC 18:1-18:1/18:0-18:2 & Alanine \\
PC 17:0-20:4 & Arginine \\
PC 18:0-20:4 & Citrulline \\
PC 18:1-22:6 & Glutamine \\
PE 16:1-18:1/16:0-18:2 & Glutamate \\
PE 16:0-18:1 & Histidine \\
PE 16:0-20:4 & Homoserine \\
PE 18:1-18:2 & Lysine \\
PE 18:1-18:1/18:0-18:2 & Serine \\
FA 14:1 Myristoleic acid* & Threonine \\
FA 14:0 Myristic acid & Tryptophan \\
FA 15:0 Pentadecylic acid* & Tyrosine \\
FA 16:1 Palmitoleic acid & Dimethylglycine \\
FA 17:1 10-Heptadecanoic acid & Taurine \\
FA 21:0 Heneicosanoic acid* & Kynurenine \\
FA 22:1 (n-9) Erucic acid* & Phosphocholine* \\
FA 23:0 Tricosanoic acid & \\
FA 24:1 (n-9) Nervonic acid & \\
FA 24:0 Lignoceric acid & \\
FA 25:0 Pentacosanoic acid & \\
FA 27:0 Heptacosanoic acid* & \\
\hline
\end{tabular}

*Detected by one extraction method only 
Table 3

List of metabolites detected only from dried serum spot (DSS) or dried whole blood spot (DWBS) by supercr

\begin{tabular}{ll}
\hline Detected only from DSS & Detected only from DWBS \\
\hline LPC 17:1 $(s n-2)$ & LPE 22:6 $(s n-1)$ \\
LPC 19:0 $(s n-1)$ & LPE_22-6 $(s n-2)$ \\
& PE 16:0-16:1/14:0-18:1 \\
& PE 16:0-16:0 \\
& PE 16:0p-18:1 \\
& PE 16:1-18:2/16:0-18:3 \\
& PE 16:0p-20:5 \\
& PE 18:0p-18:1 \\
& PE 17:0-18:1 \\
& PE 18:0e-18:1 \\
& PE 18:2-18:3 \\
& PE 16:0-20:5 \\
& PE 16:0-22:4 \\
& PE 18:1-20:3/18:2-20:2 \\
& AC 18:0 \\
& AC 18:1 \\
AC 18:2
\end{tabular}

